\title{
Evaluation of Salivary Total Antioxidant Capacity in Smokers with Severe Chronic Periodontitis
}

\author{
Masoomeh Shirzaiy, ${ }^{1}$ Mohammad Aiub Rigi Ladiz, ${ }^{1}$ Zohreh Dalirsani, ${ }^{2,}$ Javid Dehghan Haghighi, ${ }^{3}$ and \\ Alireza Nakhaii ${ }^{4}$ \\ ${ }^{1}$ Oral and Dental Disease Research Center, Zahedan University of Medical Sciences, Zahedan, IR Iran \\ ${ }^{2}$ Oral and Maxillofacial Disease Research Center, Mashhad University of Medical Sciences, Mashhad, IR Iran \\ ${ }^{3}$ Department of Community Medicine, School of Medicine, Zahedan University of Medical Sciences, Zahedan, IR Iran \\ ${ }^{4}$ Department of Biochemistry.Zahedan University of Medical Sciences, Zahedan, IR Iran \\ "Corresponding author: Zohreh Dalirsani,Oral and Maxillofacial Diseases Research Center, Mashhad University of Medical Sciences, Postal code: 91735 , Mashhad, IR Iran. Tel: \\ +98-9155002857, Fax: +98-5138829500, E-mail:zdalirsani@gmail.com
}

Received 2015 July 06; Revised 2016 October 15; Accepted 2017 January 08.

\begin{abstract}
Background: Smoking leads to changes in immune reaction and total antioxidant capacity. Smoking, through dysfunction of antioxidant system, plays an important role in the pathogenesis of inflammatory diseases.

Objectives: The current study aimed at comparing salivary antioxidant capacity in smokers and non-smokers with severe chronic periodontitis.

Patients and Methods: In this case-control study, among patients referred to Zahedan School of Dentistry without any systemic diseases, 64 patients with severe chronic periodontitis, including 27 smokers as the case group and 37 non-smokers as the control group, were selected. Stimulated salivary samples were collected and total antioxidant levels were evaluated through the Ferric reducing antioxidant power (FRAP) technique. All analyses were performed by SPSS (Ver.18) using Student's t-test, Mann Whitney test or analysis of variance (ANOVA) with significance level of 0.05 in all of the tests.

Results: Total antioxidant capacity in smokers $(378.43 \pm 207.34 \mu \mathrm{M})$ with severe chronic periodontitis was significantly lower than non-smokers $(698.30 \pm 231.86 \mu \mathrm{M})(\mathrm{P}=0.0001)$. Smoking at a rate of more than 10 cigarettes per day caused a significant decrease in total antioxidant capacity as compared to less than 10 cigarettes a day $(\mathrm{P}=0.009)$, although there was no significant relationship between total antioxidant capacity and duration of smoking $(\mathrm{P}=0.23)$.

Conclusion: smoking has an influence on antioxidant status in patients with severe chronic periodontitis. Smoking could be effective in development and progression of inflammation process through changes in oxidative stress. These findings confirm the benefits of smoking-cessation in improvement of antioxidant levels in periodontitis.
\end{abstract}

Keywords: Smoking, Periodontitis, Antioxidants, Saliva

\section{Background}

It has been confirmed that there is a direct relationship between oxidative stress and pathologic conditions or diseases. Assessment of the elements effective in oxidative stress on body's fluids, such as saliva, could be beneficial for monitoring of progression and treatment of some diseases (1).

Smoking produces large amounts of reactive oxygen species (ROS), which have an influence on normal cellular function and causes changes in the inflammation markers (2). Oxidative stress, which is produced through serious imbalance between the generation of reactive oxygen species and antioxidant protection, is effective in the pathogenesis of inflammatory conditions, such as periodontal diseases (3). On the other hand, periodontitis is a chronic infective disease, in which local and systemic factors, including diabetes and smoking change response of the immune system to local agents, such as dental plaque, and consequently affect the progression of the disease (4, 5).

Studies revealed that smoking is the second major risk factor for periodontal diseases; the possibility of chronic periodontitis in smokers is 4 times more than non-smokers (6). Smoking makes the periodontal tissue respond atypically to microbial pathogens (5). It seems that smoking plays an important role in the pathogenesis of periodontal diseases through an increase in oxidative stress, induction of an imbalance between oxidative stress and antioxidant capacity and dysregulation of innate and adaptive immune system (7).

Some researchers have paid attention to evaluate effects of cigarette on development and progression of this disease via assessment of oxidative stress in various biofluids. 
Different studies have measured anti-oxidant levels in smokers. Abdolsamadi's study demonstrated that salivary superoxide dismutase enzymes (SODs) and Glutathione Peroxidase (GSH-Px), an antioxidative parameter, in smokers are less than non-smokers, although there was not any difference between 2 groups, according to salivary uric acid levels (8). In another study, Malondialdehyde (MDA) levels were significantly higher in smokers with periodontitis as compared to non-smokers. Patients with periodontitis had more salivary lipid peroxidation than healthy controls (7).

It was determined that tobacco abuse affects the development of periodontal disease through free radicals and other reactive particles, which destroy cellular structures.

One study compared oxidative stress markers in male smokers and non-smokers with chronic periodontitis and revealed that smokers with periodontitis had concurrently higher periodontal clinical parameters and higher systemic oxidative stress (9).

Also, another study demonstrated that serum total oxidant status levels in smokers and non-smokers were reduced after periodontal treatment; however, there was no significant difference in serum total antioxidant status (TAS) levels in both groups after treatment. Also, this study showed that total antioxidant status of gingival crevicular fluid (GCF) in smokers did not show any significant difference after treatment, yet TAS levels were raised in the nonsmoker group after periodontal treatment. These results indicate differences between different body fluids regarding the oxidative/antioxidative system (10).

Furthermore, studies confirmed that smoking has a dose-dependent effect on oxidative parameters. As indicated by Agnihotri's study, which compared average levels of superoxide dismutase (SOD) in the GCF and saliva of smokers and non-smokers with periodontitis, there was a significant reduction in superoxide dismutase levels in smokers compared to non-smoker subjects. Moreover, it was proved that there was a gradual decrease in the levels of SOD of the GCF and saliva from light smoking to heavy smoking (11).

Review of previous studies suggests that assessment of oxidative stress plays an important role in evaluation of destructive effects of smoking on various body tissues.

\section{Objectives}

To the best of our knowledge, in Iran, similar studies have not been done on antioxidant levels in smokers with periodontitis. As cultural and racial factors are important elements in smoking habit and periodontal diseases, this study aim to evaluate total salivary antioxidant of smokers and non-smokers with periodontitis in South-East of Iran.

\section{Patients and Methods}

\subsection{Subject Selection}

Sixty-four patients with severe chronic periodontitis were enrolled in this descriptive-analytic case-control study. All subjects were selected among patients, who referred to Zahedan school of dentistry for dental care. Among them, patients, who were older than 30 years old and had confirmed periodontist, were divided to 2 groups: 27 smokers (case group) and 37 non-smokers (control group), both groups were matched, according to age and severity of periodontal diseases.

Inclusion criteria included:

1. Severe periodontitis with attachment loss $\geq 5 \mathrm{~mm}$ in at least $30 \%$ of teeth,

2. Gingival bleeding during gentle probing, which indicated active periodontal disease (12).

Exclusion criteria included:

1. Pregnancy

2. Alcoholism or any drug addiction, including opioids, smokeless tobacco, etc. during the past 2 years

3. Using antioxidant drugs during in the past 3 months (13) (13)

4. History of trauma or surgery during the past 4 weeks

5. Auto-immune diseases, such as arthritis rheumatoid, Sjögren's syndrome, etc. (13)

6. AIDS/HIV (13)

7. Inflammatory conditions

8. Juvenile periodontitis

9. Malignancy

10. Oral diseases, including ulcers, red and white plaques, tumors, etc.

Subjects, who smoke at least 3 cigarettes per day for at least 6 months, were included in the case group and people, who did not have any history of smoking, were included in the control group. People, who gave up smoking, were excluded from the study.

The protocol of this study was approved by the ethics panel of Zahedan University of Medical Sciences. Moreover, the subject's rights were protected and an informed consent was obtained from the study participants.

Firstly, all subjects were placed under precise periodontal examination by a periodontist and periodontal pocket depth was measured using a tiny probe (PCP-UNC 15, Hu Friedy, Leimen, Germany). Clinical attachment loss and bleeding on probing were evaluated. Information about the subjects' periodontal condition and smoking habit was recorded in the study check-list. 


\subsection{Salivary Sample Collection}

Saliva sampling was performed according to the standard technique at 9 to $11 \mathrm{am}$. Subjects were asked not to eat, drink, smoke, brush or use any hygienic device during at least 90 minutes before sample collection (14). Stimulated saliva was collected after chewing tasteless, sugarless gum for 1 minute (15). The patients were asked to bend their heads forward and spit the salvia into a 50-mL centrifuge tube without swallowing.

The saliva samples were sent to the biochemical laboratory while they were kept on dry ice. In the laboratory environment, their temperature was raised to $4^{\circ} \mathrm{C}$. Next, the saliva samples were centrifuged ( $4000 \mathrm{rpm}, 10$ minutes) to separate the cells and debris.

The samples were kept at $-80^{\circ} \mathrm{C}$ until the analysis time. This temperature is necessary for preventing the salivary proteins from the degeneration.

For evaluation of total antioxidant capacity, the samples were washed with distilled water and $10 \%$ nitric acid solution. Acetate buffer was prepared through mixing 16 $\mathrm{mL}$ of acetic acid and 3.1 grams of sodium acetate. The solution volume was then elevated to one liter and its $\mathrm{pH}$ was set to 3.6 by adding distilled water. The prepared solution included a mixture of iron chloride $(2.5 \mathrm{~mL})$ and 2, 4, 6,tripyridyl-s-triazine (TPTZ) $(2.5 \mathrm{~mL})$ added to acetate buffer (25 mL) (16).

The antioxidant capacity of saliva samples were analyzed by Ferric reducing antioxidant power(FRAP) assay using ENGLAN ultropec 3000 biotech and pharmacia spectrophotometer. In this method, the total antioxidant capacity of saliva was assessed by the ability of saliva for reduction of ferric $(\mathrm{Fe}+3)$-to-fcerrous $(\mathrm{Fe}+2)$ in the presence of a (Fe+2)-stabilizing legend, such as TPTZ. The Fe+3-TPTZ complex could change to $\mathrm{Fe}+2-\mathrm{TPTZ}$ by a reducing agent in the acidic environment. The Fe+2-TPTZ could be observed at $593 \mathrm{~nm}$ wavelength because of its intense blue color. The blue color could show the speed of production of Fe2-TPTZ complex and reducing ability of salivary samples $(16,17)$.

At the beginning of the reaction, $30 \mu \mathrm{L}$ of saliva, which had been centrifuged at $4^{\circ} \mathrm{C}$, was added to the solution. The rate of absorption was evaluated at the start of the reaction and 8 minutes later.

The difference between primary and final absorption was assessed by a standard curve. During the laboratory process, temperature of the environment was $37^{\circ} \mathrm{C}$.

\subsection{Statistical Analysis}

The data were entered in the SPSS software (Ver.18). The analyses were conducted using 3 appropriate tests: independent $t$ test, Man Whitney or analysis of variance (ANOVA) test. The significance level was set at 0.05 in all tests.

\section{Results}

Sixty four patients with severe chronic periodontitis, including 27 smokers ( 23 males and 4 females) and 37 nonsmokers (13 males and 24 females) were enrolled in this study. The average age of the case and control group was $43.40 \pm 8.13$ and $43.28 \pm 6.34$, respectively. There was no significant difference between the 2 groups ( $t$ test; $\mathrm{P}=0.942$ ).

Although the percentage of bleeding on probing (BOP) in smokers was more than non-smokers, the difference was not significant in the 2 groups ( $P>0.05)$. The BOP in smokers was $95.01 \pm 5.14$ (mean $\pm \mathrm{SD}$ ) while in non-smokers this was $93.05 \pm 20.11$. Also, there was no significant difference between the 2 groups regarding probing pocket depth (PPD) ( $\mathrm{P}>0.05)$; the mean of PPD was greater in smokers compared to non-smokers $(5.62 \pm 0.23 \mathrm{~mm}$ and $5.20 \pm 0.12 \mathrm{~mm}$, respectively) $(\mathrm{P}>0.05)$.

Total antioxidant capacity of stimulated saliva in the case group was determined in this study:

The TAOC in smokers was $378.43 \pm 207.34 \mu \mathrm{M}$ and in non-smokers, this was $698.30 \pm 231.86 \mu \mathrm{M}$. The difference was significant (t test; $\mathrm{P}=0.0001$ ) (Table 1 ).

Table 1. Demographic and Clinical Data of the Smoker and Non-Smokers

\begin{tabular}{lcc}
\hline Periodontitis Patients & Non-Smoker $(\mathbf{n}=\mathbf{3 7})$ & Smoker $(\mathbf{n}=\mathbf{2 7})$ \\
\hline Gender $(\mathbf{m} / \mathbf{f})$ & $13: 24$ & $23: 4$ \\
\hline Average age, $\mathbf{y}$ & $41.2 \pm 6.33$ & $43.6 \pm 7.23$ \\
\hline Smoking, cigarettes per day & 0 & $15.2 \pm 5.3$ \\
\hline
\end{tabular}

There was no statistically significant association between salivary TAOC and duration of smoking habit (ANOVA; $\mathrm{P}=0.235$ ) (Table 2); however, total salivary antioxidants in patients with periodontitis, who smoked more than 10 cigarettes a day, was significantly less than patients, who smoked up to 10 cigarettes per day ( $t$ test: $\mathrm{P}=0.009$ ) (Table 3).

Table 2. Comparison of Total Salivary Antioxidant According to Duration of Smoking Habit

\begin{tabular}{lccc}
\hline $\begin{array}{l}\text { Duration of } \\
\text { Smoking Habit }\end{array}$ & Number & Mean \pm SD, $\mu \mathrm{M}$ & PValue \\
\hline $\begin{array}{l}\text { Less than 6 years } \\
\text { old }\end{array}$ & 11 & $461.18 \pm 175.77$ & \\
\hline $\begin{array}{l}\text { Between 6 and 12 } \\
\text { years old }\end{array}$ & 10 & $319.99 \pm 123.07$ & 0.235 \\
\hline $\begin{array}{l}\text { More than 12 } \\
\text { years old }\end{array}$ & 6 & $324.13 \pm 330.24$ & \\
\hline
\end{tabular}

Although TAOC in 30- to 45-year-old subjects, including smokers and non-smokers $(566.26 \pm 249.23 \mu \mathrm{M})$ was 
Table 3. Comparison of Total Salivary Antioxidant According to the Number of Cigarettes Smoked Daily

\begin{tabular}{lccccc}
\hline The number of Cigarettes Per Day & Number & Median, $\mu \mathbf{M}$ & \multicolumn{2}{c}{ Percentiles } & P Value \\
\cline { 3 - 5 } & & & 25th & 50th & 0.009 \\
\hline Less than 10 cigarettes & 13 & 468.00 & 359.83 & 553.67 \\
More than 10 cigarettes & 14 & 243.10 & 113.51 & 390.08 \\
\hline
\end{tabular}

higher than subjects older than 45 years old (522.44 \pm $312.80 \mu \mathrm{M})$, there was no significant difference between subjects younger and older than 45 years old, according to total salivary antioxidant ( $\mathrm{t}$ test: $\mathrm{P}=0.84$ ). Moreover, among both age groups, non-smokers had higher total salivary antioxidant level than coeval smokers ( $t$ test: $\mathrm{P}=0.001$ and $\mathrm{P}=0.003$; respectively for $\leq 45$ and $>45$ years old) (Table 4$)$.

Table 4. Comparison of Total Salivary Antioxidant in Smokers and Non-Smokers According to Age

\begin{tabular}{lccc}
\hline Age & Number & $\begin{array}{c}\text { Mean } \pm \text { SD, } \mu \mathrm{M} \\
(\mathrm{TAOC})\end{array}$ & PValue \\
\hline 30 - 45 smoker & 17 & $403.56 \pm 167.7$ & 0.001 \\
$\begin{array}{l}\text { Nonsmoker } \\
\begin{array}{l}\text { Higher than 45 } \\
\text { Smoker }\end{array}\end{array}$ & 23 & $699.37 \pm 226.58$ & \\
\begin{tabular}{l} 
Nonsmoker \\
\hline
\end{tabular} & 10 & $328.18 \pm 275.13$ & \\
\hline
\end{tabular}

The TAOC in the 36 evaluated males was $498.96 \pm 258.18$ $\mu \mathrm{M}$ and in the 28 females was $645.88 \pm 71.71 \mu \mathrm{M}$. The analysis showed that levels of total salivary antioxidants in male subjects were significantly lower than female subjects ( $t$ test; $\mathrm{P}=0.035$ ), although this result could be related to higher number of male smokers compared to female smokers. In each gender, there was a significant difference between smokers and non-smokers according to TAOC ( $t$ test: $\mathrm{P}=0.001$ and $\mathrm{P}=0.02$; respectively for males and females) (Table 5).

Table 5. Comparison of Total Salivary Antioxidant in Smokers and Non-Smokers According to Gender

\begin{tabular}{lccc}
\hline Gender & Number & $\begin{array}{c}\text { Mean } \pm \text { SD, } \mu \mathrm{M} \\
(\mathrm{TAOC})\end{array}$ & P Value \\
\hline Male Smoker & 23 & $379.2 \pm 222.45$ & 0.001 \\
\hline Nonsmoker & 13 & $710.86 \pm 166.01$ & \\
\hline Female Smoker & 4 & $374.02 \pm 98.36$ & 0.02 \\
\hline Nonsmoker & 24 & $691.2 \pm 265.23$ & \\
\hline
\end{tabular}

\section{Discussion}

In this study, total salivary antioxidant levels of smokers and non-smokers with periodontitis were assessed.

Smoking and periodontitis compromised the antioxidant capacity of saliva and other body fluids. They increased enzymes related to oxidative stress.

Antioxidant agents could be categorized to 3 groups: one group prevents the formation of free radicals; the second group is radical-scavenging antioxidants, which eliminate free radicals and consequently inhibit destruction related to free radicals; and the third group includes DNA repairing enzymes (18).

After increased production of ROS in the oxidative stress process, higher levels of free-radical-scavenging antioxidants are consumed, therefore, researchers assess components of antioxidant activities for evaluation of oxidative stress $(19,20)$.

Although assessment of salivary total antioxidants activity is not a relevant indicator of antioxidant activities of body fluids, measurement of several antioxidants is timewasting, complicated and expensive; as a result, TAOC index, which shows accumulative effect of all antioxidants in the bio-fluids, is assumed to be an appropriate indicator $(21,22)$.

Antioxidants activities are modified through variant factors, including nutrition, stress, physical activity and smoking. In fact, smoking leads to an imbalance between reactive oxygen species and antioxidant levels and consequently induces oxidative stress (23).

On the other hand, smoking is one of the most important factors in development and progression of periodontal diseases; prevalence of periodontitis in cigarette smokers is 6 to 6.2 times more than non-smokers. Nicotine changes immune reaction, gingival inflammation, and oxidant and antioxidant capacity $(7,24)$. Several studies about oxidant and antioxidant activity and effective factors (such as Reactive Oxygen Species and lipid peroxidation products) have been done, which emphasized that saliva is useful in the diagnosis of conditions, which affect oxidant-antioxidant system, such as periodontal diseases and pre-malignant lesions.

In the present study, salivary total antioxidant capac- 
ity of smokers was significantly lower than non-smokers. This result was similar to Guentsch's study, which revealed that antioxidant levels of smokers involved with periodontitis were lower than non-smoker subjects, while MDA levels (the last product of oxidative stress) were considerably higher in smoker subjects than non-smokers (7). Their study confirmed that smoking leads to increased oxidative stress, as well as an imbalance between oxidants and antioxidants (7). Also, Rai et al. concluded that total glutathione of saliva was significantly higher in smokers with periodontitis in comparison with non-smokers (25). They demonstrated that salivary total glutathione concentrations were significantly decreased after some periodontal treatments, such as scaling and root planning in smokers as well as in nonsmokers involved with periodontitis (25). There were some similarities according to study subjects in Rai's study and the present study, yet in the current study the entire salivary antioxidant capacity was evaluated, which was lower in smokers than non-smokers involved with periodontitis. Furthermore, Abdolsamadi et al. with evaluation of antioxidant concentrations in healthy smokers and non-smokers revealed that there was a decrease in salivary antioxidant levels in smokers (8). This result was comparable with the present study; however, in the current study all subjects were involved with periodontitis. Moreover, Ahmad's study showed a significant decrease in some salivary antioxidant factors in smokers as compared to non-smokers. They concluded that salivary antioxidants are relevant factors for evaluation of oxidative stress in smokers. Also, there was an indirect correlation between salivary antioxidant levels and duration of smoking habit (26), although in the present study, duration of habit did not have any significant influence on salivary antioxidant capacity.

There is some controversy between the results of different studies regarding the levels of various elements and enzymes related to oxidative stress. Diken's study revealed that the activity of glutathione in erythrocytes was not influenced by smoking (27). Moreover, Zappacosta et al. demonstrated that smoking causes a decrease in concentration of salivary glutathione; however, they found that there was no significant difference between smokers and non-smokers regarding uric acid concentrations and total antioxidant capacity (28). On the other hand, Reddy et al. revealed a significant decrease in salivary and serum superoxide dismutase enzymes in smokers as compared to non-smokers (29). Also, Kanehira et al. concluded that concentrations of salivary thiocyanate and copper/zinc superoxide dismutase were enhanced in smokers while levels of other enzymes, such as peroxidase and glutathione peroxidase, were lower in these subjects (30). However, this study evaluated these elements in only elderly people in contrast to the current study.

It seems that with aging, oxidative stress increases while antioxidants activity, which plays an important role in some inflammatory diseases, does not show any significant difference (31). In agreement with this theory, Balkan et al. revealed that antioxidant activities in elderly subjects (61 to 85 years old) were lower than young people (32).

Antioxidant system is very complicated and includes different types of extra and intra-cellular molecules and enzymes in biofluids, such as plasma and saliva.

In some studies, antioxidant capacity of serum and gingival crevicular fluid in smokers and cases with periodontitis were assessed $(23,33)$. Serum and gingival crevicular fluid TAOC in smokers and non-smokers involved with periodontitis were estimated in one study, which revealed that gingival crevicular fluid and serum TAOC decrease in smokers as compared to non-smokers (23), the results of that study was similar to the present study in which smokers had lower levels of TAOC in comparison with nonsmokers; however, different body fluids were evaluated in these two studies.

In another study, Toker et al. evaluated the effects of smoking on interleukin-1 $\beta$ levels and total oxidant and antioxidant levels of gingival crevicular in periodontal diseases. The interleukin-1 $\beta$ levels in gingival crevicular fluid were significantly higher in smokers than non-smokers (3).

These conflicting results of studies could be related to a variety in sample sizes, the number of cigarettes per day, type and duration of smoking habit, assessment of various antioxidant elements in different studies, various kits or laboratory techniques, different structure of the studies and different age groups and other properties of subjects. It seems that according to most studies' results, smoking changes antioxidant capacity, yet the mechanism of these alterations have not been well known (34).

There is a challenge about the exact correlation between smoking, periodontitis, and oxidative stress. Smoking could affect the antioxidant system through different mechanisms. Smoking causes an increase in oxidative stress and production of free radicals with stimulation of interleukin- $1 \beta$. In oxidative stress, an imbalance between production of free radicals and antioxidant levels leads to an increase in ROS levels in the biological environment. Overproduction of ROS causes destruction of some bio-molecules, including DNA, amino acids, carbohydrates, lipids, and disturbance in structural organization and cell function (35-37).

Bakhtiari et al. confirmed that oxidative stress from smoking was not reduced even after using anti-oxidant drugs, including vitamin C (38). On the other hand, in an animal study, the activities of some antioxidant enzymes, including superoxide dismutase, catalase, and glutathione 
peroxidase activities, were increased in rats exposed to cigarette smoke. The mentioned study showed that using supplementation of antioxidants before smoking attenuated the harmful effects of cigarette on the pulmonary system; however, it was not enough to protect the organs completely (39).

The current study revealed that salivary TAOC was higher in female than male subjects. Brock's study showed that salivary antioxidants capacity in healthy males was greater than females; however, there was no difference according to gender in patients with periodontal diseases. In contrast, GCF antioxidant concentrations in males were lower than females in both healthy and patient groups; however, the difference was not significant (15). In another study, Scully confirmed that mean levels of total antioxidant capacity were significantly lower in females as compared to males, regardless of periodontal problem, although, they revealed that periodontal diseases was associated with a decrease in salivary antioxidant capacity (17). It seems that some conditions, such as periodontitis, could change antioxidants capacity in both genders.

Various results of these studies could be related to simultaneous presence of periodontitis and smoking in our study's subjects. Furthermore, fewer female smokers enrolled in the current study in comparison with male smokers had some influence on the results.

Moreover, in the present study, salivary TAOC in subject, who smoked more than 10 cigarettes per day was lower than other smokers. It is logical to claim that harmful effects of smoking on antioxidant system are based on daily dose. Review of other studies showed that smoking is a major risk factor for acceleraion of periodontal diseases, and this effect is dose-dependent $(40,41)$.

Briefly, it is logical to claim that smoking could effect oxidative-anti oxidative system in patients with periodontitis, although the exact mechanism is not well known.

\subsection{Conclusions}

In the present study, among subjects involved with periodontitis, smoking caused some reduction in antioxidant capacity. This effect of smoking on antioxidant activity was dose-dependent. In respect to effects of cigarette on oxidative stress, smoking-cessation efforts could be effective in treatment of periodontal diseases through an increase in antioxidant capacity.

\section{Acknowledgments}

The authors would like to extend their appreciation to the vice chancellor for research, Zahedan University of Medical Sciences for their financial support. "The results described in this paper are part of a (D.D.S) student thesis proposal".

\section{Footnotes}

Authors' Contribution: Design, study concept and supervision: Masoomeh Shirzaiy; Acquisition of data and administrative, technical, and material support: Mohammad Aiub Rigi Ladiz; Analysis and interpretation of data: Javid Dehghan Haghighi; Drafting of the manuscript and critical revision of the manuscript for important intellectual content: Zohreh Dalirsani.

Conflict of Interest: The authors declare no conflicts of interest in this work.

\section{References}

1. Golusinska-Kardach E, Napierala M, Sokalski J, Kardachi H, Florek E. [Periodonta disease in smokers, and the parameters of oxidative stress]. Przegl Lek. 2015;72(10):584-7. [PubMed: 26946573].

2. Niu J, Wang K, Kolattukudy PE. Cerium oxide nanoparticles inhibit oxidative stress and nuclear factor-kappaB activation in H9c2 cardiomyocytes exposed to cigarette smoke extract.J Pharmacol Exp Ther. 2011;338(1):53-61. doi: 10.1124/jpet.111.179978. [PubMed: 21464334].

3. Toker H, Akpinar A, Aydin H, Poyraz O. Influence of smoking on interleukin-1beta level, oxidant status and antioxidant status in gingival crevicular fluid from chronic periodontitis patients before and after periodontal treatment. J Periodontal Res. 2012;47(5):572-7. doi: 10.1111/j.1600-0765.2012.01468.x. [PubMed: 22364664].

4. Kim SC, Kim OS, Kim OJ, Kim YJ, Chung HJ. Antioxidant profile of whole saliva after scaling and root planing in periodontal disease. J Periodontal Implant Sci. 2010;40(4):164-71. doi: 10.5051/jpis.2010.40.4.164. [PubMed: 20827325].

5. Buduneli N, Kinane DF. Host-derived diagnostic markers related to soft tissue destruction and bone degradation in periodontitis. J Clin Periodontol. 2011;38 Suppl 11:85-105. doi: 10.1111/j.1600051X.2010.01670.x. [PubMed: 21323706].

6. Haber J, Wattles J, Crowley M, Mandell R, Joshipura K, Kent RL. Evidence for cigarette smoking as a major risk factor for periodontitis. J Periodontol. 1993;64(1):16-23. doi: 10.1902/jop.1993.64.1.16. [PubMed: 8426285].

7. Guentsch A, Preshaw PM, Bremer-Streck S, Klinger G, Glockmann E, Sigusch BW. Lipid peroxidation and antioxidant activity in saliva of periodontitis patients: effect of smoking and periodontal treatment. Clin Oral Investig. 2008;12(4):345-52. doi: 10.1007/s00784-008-0202-z. [PubMed: 18509684].

8. Abdolsamadi HR, Goodarzi MT, Mortazavi H, Robati M, AhmadiMotemaye F. Comparison of salivary antioxidants in healthy smoking and non-smoking men. Chang Gung Med J. 2011;34(6):607-11. [PubMed: 22196063].

9. Aziz AS, Kalekar MG, Suryakar AN, Benjamin T, Prakashan MJ, Ahmed BM, et al. Assessment of some biochemical oxidative stress markers in male smokers with chronic periodontitis. Indian J Clin Biochem. 2013;28(4):374-80. doi: 10.1007/s12291-012-0283-y. [PubMed: 24426240].

10. Akpinar A, Toker H, Ozdemir H, Bostanci V, Aydin H. The effects of nonsurgical periodontal therapy on oxidant and anti-oxidant status in smokers with chronic periodontitis. Arch Oral Biol. 2013;58(6):717-23. doi: 10.1016/j.archoralbio.2012.11.009. [PubMed: 23261252]. 
11. Agnihotri R, Pandurang P, Kamath SU, Goyal R, Ballal S, Shanbhogue AY, et al. Association of cigarette smoking with superoxide dismutase enzyme levels in subjects with chronic periodontitis. J Periodontol. 2009;80(4):657-62. doi: 10.1902/jop.2009.080545. [PubMed: 19335086].

12. Page RC, Eke PI. Case definitions for use in population-based surveillance of periodontitis. J Periodontol. 2007;78(7 Suppl):1387-99. doi: 10.1902/jop.2007.060264. [PubMed:17608611].

13. Shirzad A, Pouramir M, Seyedmajidi M, Jenabian N, Bijani A, Motallebnejad M. Salivary total antioxidant capacity and lipid peroxidation in patients with erosive oral lichen planus. J Dent Res Dent Clin Dent Prospects. 2014;8(1):35-9. doi: 10.5681/joddd.2014.006. [PubMed: 25024837].

14. Navazesh M. Methods for collecting saliva. Ann N Y Acad Sci. 1993;694:72-7. [PubMed: 8215087].

15. Brock GR, Butterworth CJ, Matthews JB, Chapple IL. Local and systemic total antioxidant capacity in periodontitis and health.J Clin Periodontol. 2004;31(7):515-21. doi: 10.1111/j.1600-051X.2004.00509.x. [PubMed: 15191586].

16. Azizi A, Sarlati F, Parchakani A, Alirezaei S. Evaluation of Whole Saliva Antioxidant Capacity in Patients with Periodontal Diseases. Open J Stomatol. 2014;04(04):228-31. doi: 10.4236/ojst.2014.44031.

17. Sculley DV, Langley-Evans SC. Periodontal disease is associated with lower antioxidant capacity in whole saliva and evidence of increased protein oxidation. Clin Sci (Lond). 2003;105(2):167-72. doi: 10.1042/CS20030031. [PubMed: 12650638].

18. Battino M, Bullon P, Wilson M, Newman H. Oxidative injury and inflammatory periodontal diseases: the challenge of anti-oxidants to free radicals and reactive oxygen species. Crit Rev Oral Biol Med. 1999;10(4):458-76. [PubMed: 10634583].

19. Lih-Brody L, Powell SR, Collier KP, Reddy GM, Cerchia R, Kahn E, et al. Increased oxidative stress and decreased antioxidant defenses in $\mathrm{mu}$ cosa of inflammatory bowel disease. Dig Dis Sci. 1996;41(10):2078-86. [PubMed: 8888724].

20. Hoelzl C, Bichler J, Ferk F, Simic T, Nersesyan A, Elbling L, et al. Methods for the detection of antioxidants which prevent age related diseases: a critical review with particular emphasis on human intervention studies. J Physiol Pharmacol. 2005;56 Suppl 2:49-64. [PubMed: 16077190].

21. Suresh DR, Annam V, Pratibha K, Prasad BV. Total antioxidant capacity-a novel early bio-chemical marker of oxidative stress in HIV infected individuals. J Biomed Sci. 2009;16:61. doi: 10.1186/1423-0127-1661. [PubMed: 19583866].

22. Battino M, Ferreiro MS, Gallardo I, Newman HN, Bullon P. The antioxidant capacity of saliva. JClin Periodontol. 2002;29(3):189-94. [PubMed: 11940135].

23. Mohan TSA, Theagarayan P. Evaluation of total antioxidant levels in smokers and non-smokers with chronic periodontitis. SRM J Res Dent Sci. 2011;2(1):9.

24. Research . Position paper: tobacco use and the periodontal patient. Research, Science and Therapy Committee of the American Academy of Periodontology. J Periodontol. 1999;70(11):1419-27. doi 10.1902/jop.1999.70.11.1419. [PubMed:10588507].

25. Rai B. Total salivary glutathione levels: Periodontitis in smoker and non-smoker. Adv Med Dent Sci. 2008:47-50.

26. Ahmed AM. Salivary Antioxidants Superoxide Dismutase and Glutathione Peroxidase in smokers comparing to Non-smokers. Bio Sci Res. 2013;4:4-9.
27. Diken H, Kelle M, Tumer C, Deniz B, Baylan Y, Sermet A. Effects of cigarette smoking on blood antioxidant status in short-term and long-term smokers. TurkJ Med Sci. 2001;31(6):553-8.

28. Zappacosta B, Persichilli S, De Sole P, Mordente A, Giardina B. Effect of smoking one cigarette on antioxidant metabolites in the saliva of healthy smokers. Arch Oral Biol. 1999;44(6):485-8. [PubMed: 10401526].

29. Reddy S, Swapna LA, Ramesh T, Singh TR, Pradeep K. Influence of cigarette smoking on blood and salivary super oxide dismutase levels among smokers and non-smokers. J Investig Clin Dent. 2012;3(4):298303. doi: 10.1111/j.2041-1626.2011.00097.x. [PubMed: 23129144].

30. Kanehira T, Shibata K, Kashiwazaki H, Inoue N, Morita M. Comparison of antioxidant enzymes in saliva of elderly smokers and non-smokers. Gerodontology. 2006;23(1):38-42. doi: 10.1111/j.17412358.2006.00077.x. [PubMed: 16433640].

31. Valko M, Leibfritz D, Moncol J, Cronin MT, Mazur M, Telser J. Free radicals and antioxidants in normal physiological functions and human disease. Int J Biochem Cell Biol. 2007;39(1):44-84. doi: 10.1016/j.biocel.2006.07.001. [PubMed:16978905].

32. Balkan J, Kanbagli O, Mehmetcik G, Mutlu-Turkoglu U, Aykac-Toker G, Uysal M. Increased lipid peroxidation in serum and low-density lipoproteins associated with aging in humans. Int $J$ Vitam Nutr Res. 2002;72(5):315-20. doi: 10.1024/0300-9831.72.5.315. [PubMed: 12463107].

33. Wei D, Zhang XL, Wang YZ, Yang CX, Chen G. Lipid peroxidation levels, total oxidant status and superoxide dismutase in serum, saliva and gingival crevicular fluid in chronic periodontitis patients before and after periodontal therapy. Aust Dent J. 2010;55(1):70-8. doi: 10.1111/j.1834-7819.2009.01123.x. [PubMed: 20415915].

34. Yildiz L, Kayaoglu N, Aksoy H. The changes of superoxide dismutase, catalase and glutathione peroxidase activities in erythrocytes of active and passive smokers. Clin Chem Lab Med. 2002;40(6):612-5. doi: 10.1515/CCLM.2002.106. [PubMed: 12211658].

35. Meucci E, Littarru C, Deli G, Luciani G, Tazza L, Littarru GP. Antioxidant status and dialysis: plasma and saliva antioxidant activity in patients with fluctuating urate levels. Free Radic Res. 1998;29(5):367-76. [PubMed: 9925029]

36. Moore S, Calder KA, Miller NJ, Rice-Evans CA. Antioxidant activity of saliva and periodontal disease. Free Radic Res. 1994;21(6):417-25. [PubMed: 7834056].

37. Hirayama O, Takagi M, Hukumoto K, Katoh S. Evaluation of antioxidant activity by chemiluminescence. Anal Biochem. 1997;247(2):23741. doi: 10.1006/abio.1997.2053. [PubMed: 9177683]

38. Bakhtiari S, Bigom Taheri J, Bakhshi M, Mortazavi H, Shah Hoseini A, Vahid Dastjerdi E, et al. Effect of vitamin C on salivary total antioxidant capacity in smokers. Iran JPharm Res. 2012;11(4):1045-9. [PubMed: 24250535].

39. Sohn HO, Lim HB, Lee YG, Lee DW, Kim YT. Effect of subchronic administration of antioxidants against cigarette smoke exposure in rats. Arch Toxicol. 1993;67(10):667-73. [PubMed: 8135656].

40. Ho YC, Chang YC. Regulation of nicotine-induced cyclooxygenase2 protein expression in human gingival fibroblasts. Acta Pharmacol Sin. 2006;27(4):409-13. doi: 10.1111/j.1745-7254.2006.00286.x. [PubMed: 16539839].

41. Rao P, Ande A, Sinha N, Kumar A, Kumar S. Effects of Cigarette Smoke Condensate on Oxidative Stress, Apoptotic Cell Death, and HIV Replication in Human Monocytic Cells. PLoS One. 2016;11(5):e0155791. doi: 10.1371/journal.pone.0155791. [PubMed: 27203850] 